\title{
O Efeito do Status de Sobrevivência sobre Gastos com Internações Hospitalares Públicas no Brasil em uma Perspectiva Temporal
}

\author{
Cristina Guimarães Rodrigues \\ Doutora em Demografia pelo Cedeplar/UFMG e pós-doutoranda pelo Departamento de \\ Contabilidade e Atuária (FEA/USP) - Endereço para contato: Av. Prof. Luciano Gualberto 908, \\ FEA-3 - Cidade Universitária - São Paulo - CEP: 05508-010 \\ E-mail: guimaraes.cristina@gmail.com
}

\section{Luís Eduardo Afonso}

Professor do Departamento de Contabilidade e Atuária (FEAVUSP)

Endereço para contato: Av. Professor Luciano Gualberto 908, FEA-3 - Cidade Universitária

São Paulo - CEP: 05508-010

E-mail: lafonso@usp.br

Recebido em 26 de julho de 2011. Aceito em 20 de junho de 2012.

\section{Resumo}

As projeções de gastos com saúde apontam para um crescimento considerável das despesas em decorrência do envelhecimento populacional. No entanto, estudos mostram que os gastos se concentram no fim da vida e, dessa forma, projeções que não levam em conta variáveis de proximidade à morte tendem a superestimar as projeções. O objetivo deste trabalho é analisar a magnitude dos gastos com internações públicas no Brasil por status de sobrevivência, e identificar se existe uma relação entre despesas com internações para indivíduos próximos à morte e idade à morte. $\mathrm{O}$ banco de dados empregado é oriundo do Sistema de Informações Hospitalares do DATASUS - SIH/DATASUS - para o período de 1995 a 2007 no Brasil. Foi analisada a evolução dos gastos totais e a tendência dos gastos por grupo etário para aqueles pacientes que tiveram como motivo de saída do hospital a alta (sobreviventes) ou o óbito (pacientes terminais). A fim de analisar o efeito do status de sobrevivência sobre os gastos com internações no futuro, simulamos projeções de gastos em 2050, desagregados entre gastos de sobreviventes e gastos relacionados à morte. Os resultados mostram que o padrão de gastos por status de sobrevivência no Brasil é crescente por grupo etário para o grupo de sobreviventes, e decrescente para os indivíduos que faleceram. Também se verificou que a razão de gastos mortos/sobreviventes diminui com a idade. A simulação da projeção de gastos com internações para 2050 mostra que quando se considera apenas o perfil etário dos gastos médios em 2007 , há um crescimento de mais de $380 \%$ nos gastos com internações em 2050 quando comparado a 2007, mas quando os gastos são projetados segundo o status de sobrevivência, o crescimento não passa de $70 \%$.

Agradecemos ao parecerista anônimo pela leitura cuidadosa e valiosas sugestões em uma versão
anterior do manuscrito. Quaisquer erros e omissões são de inteira responsabilidade dos autores. 
Projeção retrospectiva para 2007 mostra que o efeito do envelhecimento é menor quando o efeito da proximidade à morte é levado em consideração.

\title{
Palavras-Chave
}

gastos com internação hospitalar, status de sobrevivência, Brasil

\section{Classificação JEL}

$118, \mathrm{~J} 11, \mathrm{~J} 18$

\begin{abstract}
Health care spending projections point to a considerable increase in expenditure due to population aging. However, studies show that healthcare spending is concentrated at the end of life. Therefore, expenditure projections that do not take proximity to death into account tend to be overestimated. The aim of this paper is to analyze the amount of spending on public inpatient care in Brazil by survivorship status and determine if there is a relationship between inpatient costs for individuals close to death and age at death. We used data about hospital admissions over the years 1995 to 2007 from Sistema de Informações Hospitalares do Sistema Único de Saúde-SIH/SUS (Hospital System Information from Unified Health System), an administrative record from the federal government that includes information on all public hospitalizations in Brazil. Survivorship status is given by those who survived in the year of analysis (survivors) and for those who died in the same period (decedents). We analyzed inpatient expenditure trends over time by age group for survivors and decedents, and the ratio of decedents/ survivors costs. Projections of inpatient expenditures in 2050 were performed by the multiplication of per capita inpatient costs by age by the projected number of hospitalizations in each age group. In order to analyze the effect of survival status on hospital spending, we first estimated the number of decedents and survivors in each age group in the future, and then we multiplied these numbers through the per capita expenditure by survivorship status and age. The results show that the pattern of expenditure by survivorship status in Brazil follows the pattern found in other countries: for survivors expenditures increase with age and for decedents there is a downward trend by age. The ratio decedents/survivors cost has a negative relationship with age but increases over time. Applying the age profile of the average expenditure for 2007 results in a projected increase of over $380 \%$ in spending on hospital admissions in 2050. But when expenditures are projected by survivorship status, the growth is no more than $70 \%$. A backward projection for 2007 shows the effect of ageing is lower when the effect of proximity to death is accounted for.
\end{abstract}

\section{Keywords}

public inpatient care spending, survivorship status, Brazil 


\section{Introdução}

Um dos principais desafios atuais dos sistemas de saúde refere-se ao gerenciamento dos elevados gastos associados à utilização de serviços de saúde (Fogel, 2008). Em um sistema de saúde cujas diretrizes são o provimento público e universal de serviços, como no Brasil, o crescimento nos gastos com saúde é ainda mais preocupante, visto que pode comprometer a sustentabilidade financeira de todo o sistema de saúde, com consequências sobre a oferta e qualidade dos serviços prestados à população. Pode haver ainda reflexos de grande monta sobre as finanças públicas do país de um modo geral.

A trajetória dos gastos com saúde envolve a interação de diversos fatores, tanto do lado da demanda quanto da oferta, que agem como atenuantes ou propulsores dos gastos. Sob a ótica da demanda podemos citar o envelhecimento populacional, aumento na cobertura de diversos tipos de serviços e estado de saúde da população (Raitano, 2006). O lado da oferta reflete o progresso tecnológico, inflação no setor saúde, políticas em relação ao acesso e custo dos serviços (Lee \& Miller, 2002).

Do ponto de vista da demanda, um dos fatores mais discutidos atualmente refere-se aos efeitos do envelhecimento populacional sobre os gastos. Projeções de gastos com saúde para vários países mostram que o envelhecimento pode trazer uma séria crise financeira no setor saúde (Spillman \& Lubitz, 2000; Mayhew, 2000; Dang et al., 2001; Antolin et al., 2001). Isso ocorre porque as projeções levam em consideração o padrão de gastos por idade, que possui um formato em $J$, indicando que há uma relação positiva entre idade e gastos (Nunes, 2004). Com gastos crescentes com a idade aliados à maior proporção de pessoas em idades mais velhas, os gastos com saúde tendem a representar uma parcela considerável do PIB. Para o Brasil, trabalho de Kilsztajn et al. (2003) estima que o gasto real com saúde em 2050 seria responsável por $10,7 \%$ do PIB, um crescimento de $30 \%$ em relação ao ano de 1998.

\footnotetext{
l A inflação no setor saúde inclui os subgrupos de assistência médica, medicamentos e outros produtos farmacêuticos, que sofrem variação maior de preços ocasionados, em grande parte, pela contínua incorporação tecnológica. Analisando o Índice de Preço Acumulado mensal de janeiro de 2005 a janeiro de 2010 calculado pela Fundação Instituto de Pesquisas Econômicas (FIPE), percebe-se que a inflação na saúde é maior que a inflação em outros grupos, como habitação, alimentação, transporte e vestuário.
} 
No entanto, existem dois argumentos que refutam as evidências de que o envelhecimento populacional seria o grande responsável pelo aumento nos gastos com saúde no futuro. O primeiro argumento se baseia nas mudanças no estado de saúde da população, que podem ter consequências muito distintas sobre os gastos. Um aumento na esperança de vida pode ser acompanhado por um aumento na idade média em que as doenças começam a ocorrer, e proporcionalmente maior que o aumento na esperança de vida. Estudos mostram que o período de prevalência das doenças na população está diminuindo nos países desenvolvidos (Fries, 2000; 2003), com a postergação da idade média de início das incapacidades (Fogel, 2003; 2005). Segundo Fries (2003), este fato tem como consequência uma redução na utilização de cuidado médico em cerca de $10 \%$ ao ano.

O segundo argumento tem como justificativa o fato de que o padrão de gastos com saúde é fortemente influenciado pela concentração da utilização no fim da vida (Scitovsky, 1984; Lubitz \& Riley, 1993; Zweifel et al., 1999; McGrail et al., 2000; Yang et al., 2003; Polder et al., 2006). Como os idosos apresentam as maiores probabilidades de morte, isso explicaria o motivo pelo qual os gastos aumentam com a idade, e não pela idade por si só (Yang et al., 2003; Seshamani \& Gray, 2004; Polder et al., 2006; Raitano, 2006; Layte, 2007). A associação entre proximidade à morte e o aumento nos gastos está no fato de que, para prolongar a vida, são utilizados procedimentos mais intensivos em tecnologia e, consequentemente, mais caros do que aqueles que são utilizados com a população sobrevivente (McGrail et al., 2000).

\subsection{A Relação entre Proximidade à Morte e os Gastos com Saúde}

A relação entre proximidade à morte e gastos com saúde pode ser mensurada por dois tipos de variáveis: status de sobrevivência e tempo até a morte. No primeiro caso estão sendo comparados o gasto e a utilização com serviços de saúde de dois grupos populacionais distintos, aqueles que sobreviveram e aqueles que faleceram no período de análise. Esse tipo de análise corresponde ao chamado "custo da morte" (Payne, 2007; 2009). Nesse caso considera-se, em geral, o gasto com a última utilização dentro de um período delimitado, por exemplo, um ano corrente. Para gastos hospitalares, por exemplo, são comparados os gastos efetuados na última internação para aqueles 
que tiveram como saída do hospital "alta" (sobreviventes) ou "óbito" (decedents). Para permitir a comparação entre sobreviventes e não sobreviventes em um ano corrente, os gastos dos não sobreviventes podem ser resgatados para os 12 meses anteriores à morte, enquanto os gastos dos sobreviventes são computados para o ano calendário (Payne, 2009; Andrade et al., 2011).

No segundo caso - tempo até a morte - soma-se o gasto segundo o tempo restante de vida, normalmente dado em anos ou meses. Nesse caso, acompanha-se o gasto efetuado pelos indivíduos dentro de um período determinado até o momento de sua morte. Por exemplo, se um indivíduo falece no dia 10 de julho do ano $X$, soma-se o gasto do dia 11 de julho do ano $X-1$ até o gasto em 10 de julho do ano $X$ para computar o gasto acumulado um ano antes da morte. $O$ gasto correspondente a dois anos antes da morte é computado do dia 11 de julho de $X$-2 até 10 de julho de $X$-1, e assim sucessivamente.

Os estudos que analisam os gastos por tempo até a morte mostram que os gastos crescem à medida que a morte se aproxima, principalmente no último ano de vida. Batljan \& Lagergren (2004) estimaram que menos de $1 \%$ da população com zero anos de vida restante (12 meses que antecederam à morte) contava com $11 \%$ do total de gastos com internações na Suécia de 1992 a 1997. Considerando os gastos nos últimos meses de vida, Gabriele et al. (2006) mostram que em quatro regiões da Itália, analisadas de 1999 a 2001, houve um aumento considerável nos gastos no último mês de vida, de cerca de cinco vezes o gasto nos dois meses que antecedem à morte para homens e três para as mulheres. Estimativas para a Austrália em 2002 (Calver et al., 2006) mostram que os gastos com hospitalização para aqueles no último mês de vida são $38 \%$ maiores do que para aqueles no segundo mês.

$\mathrm{Na}$ linha dos estudos que procuram analisar a dimensão dos gastos por status de sobrevivência, foca-se, geralmente, no padrão etário dos gastos a fim de identificar se há uma relação entre gastos com morte e idade à morte (idade em que a pessoa faleceu). Uma das formas de mensurar essa relação é por meio da razão de gastos óbitos/sobreviventes (Payne et al., 2009). Esses estudos mostram que a diferença no gasto per capita de sobreviventes e não sobreviventes diminui em idades mais avançadas, contribuindo para uma redução na razão de gastos óbitos/sobreviventes com a idade (Lubitz et al., 1995; McGrail 
et al., 2000; Seshamani \& Gray, 2004; Ahn et al., 2005). Isso ocorre tanto por uma tendência decrescente do gasto per capita dos não sobreviventes com a idade quanto por um aumento no gasto per capita dos sobreviventes com a idade. Uma das explicações para a redução do gasto médio dos não sobreviventes em idades mais avançadas é que há uma resistência por parte dos médicos em tratar a saúde desses pacientes da mesma forma como tratariam os mais jovens com os mesmos sintomas, em virtude da fragilidade do organismo dos idosos (Cutler \& Sheiner, 1998; Breyer \& Felder, 2006; Calver et al., 2006).

No Brasil existem poucas evidências sobre a relação entre gastos e status de sobrevivência (Berenstein, 2009; Andrade et al., 2011; Maia, 2012). Em geral, os trabalhos mostraram que, assim como encontrado na literatura internacional, os gastos per capita para aqueles pacientes próximos à morte são bem superiores ao gasto para os sobreviventes, e que esse efeito é mais relevante para os grupos etários mais jovens, tanto no setor público de saúde (Berenstein, 2009) quanto no setor privado (Andrade et al., 2011; Maia, 2012).

A análise dos gastos por status de sobrevivência é importante do ponto de vista da projeção de gastos. Os trabalhos mostram que as projeções que consideram apenas gastos médios totais por grupo etário tendem a superestimar as projeções, o que torna visível a necessidade da distinção da população em termos do status de sobrevivência (Miller, 2001; Stearns \& Norton, 2004; Polder \& Achterberg, 2004; OECD, 2005). Esse tipo de análise é importante para todo o setor saúde, e crucial quando se trata de internação hospitalar, uma vez que a assistência hospitalar consome a maior parte dos recursos assistenciais em saúde (Bogue et al., 2007).

O sistema público no Brasil é responsável pela maior parte das internações no país. Segundo informações da Pesquisa Nacional por Amostra de Domicílios (PNAD) de 2008, cerca de 68\% de todas das internações no país foram cobertas pelo sistema público. Além disso, aproximadamente $17 \%$ das pessoas com planos de saúde se internaram na rede pública. Esses fatores tornam primordial uma análise mais apurada do gasto com serviços hospitalares. Sendo assim, o objetivo deste trabalho é responder às seguintes questões para o caso brasileiro:

1. Como os gastos com internação para aqueles próximos à morte estão relacionados à idade à morte? 
2. Como essas relações se modificam em um período de 12 anos, de 1995 a 2007 ?

3. Qual o efeito dos status de sobrevivência sobre a projeção dos gastos com internações em 2050?

\section{Base de Dados e Variáveis}

A base de dados utilizada neste trabalho corresponde ao Sistema de Informações Hospitalares do SUS (SIH/SUS) entre os anos de 1995 e 2007 para todo o Brasil. O Sistema de Informações Hospitalares do Sistema Único de Saúde (SIH/SUS) foi criado em 1990 por meio de portarias ministeriais, com o objetivo de controlar e avaliar as ações de saúde, bem como estabelecer um sistema único de repasses financeiros da produção hospitalar. ${ }^{2}$ Esse sistema é gerado com base na informatização do formulário de Autorização de Internação Hospitalar (AIH), um registro administrativo dos hospitais públicos ou particulares conveniados ao SUS. A informatização desse formulário tem como objetivo gerar créditos destinados ao pagamento de qualquer procedimento realizado com os pacientes que utilizam o sistema público de saúde em caráter de internação.

A variável de gasto utilizada corresponde ao gasto total com internação (variável valor_tot), referente a todos os procedimentos utilizados na internação do paciente. Os gastos foram desagregados por sexo, grupos etários quinquenais (com intervalo aberto acima dos 80 anos) e status de sobrevivência. O status de sobrevivência é dado pela distinção da população entre aqueles que sobrevivem e falecem dentro de um determinado período de análise. Nesse caso, o que distingue a população em termos do status de sobrevivência é o motivo da saída do hospital, que pode ser por alta (sobrevivente) ou por morte (falecido). O montante total de gastos com internação entre 1995 e 2007, não deflacionado, corresponde a quase 60 bilhões de reais. Para deflacionar a série histórica de gastos, foi utilizado o deflator IPCA, subitem saúde e cuidados pessoais, tendo como ano-base o ano de 1995.

2 Portaria no 896, de 29 de junho de 1990 e Resolução INAMPS no 227 de 27 de julho de 1990. 
O total de internações de 1995 a 2007 vem se reduzindo; de 13 milhões em 1995 passou a 10 milhões em 2007. Por outro lado, a taxa de mortalidade hospitalar aumentou de 2,27\% em 1995 a 3,24\% em 2007, como mostra a FIG. 1. A taxa de mortalidade hospitalar corresponde à proporção de pessoas que tiveram saída por óbito no ano de análise. Também deve ser ressaltado que o percentual de gastos com pacientes que faleceram em relação ao total de gastos aumentou de 5,5\% em 1995 para quase 8\% em 2007.

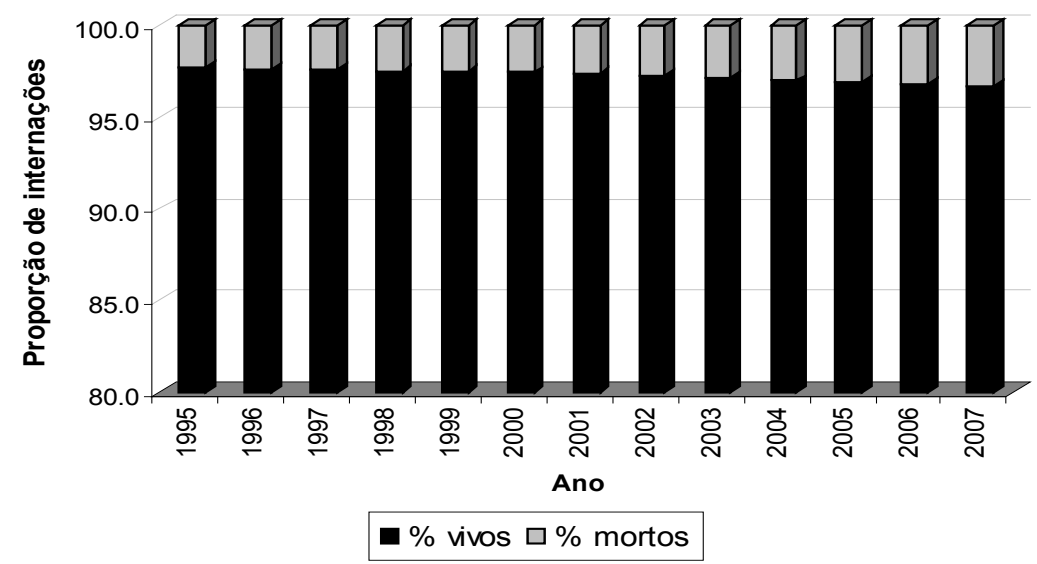

Figura 1- Proporção de internações segundo o status de sobrevivência - Brasil, 1995 a 2007

Fonte: SIH / DATASUS - 1995 a 2007.

\section{Tendência dos Gastos Totais}

A FIG. 2 apresenta o gasto per capita (em R $\$$ de 1995) por status de sobrevivência de 1995 a 2007. Observa-se que os gastos per capita com os pacientes não sobreviventes representam o dobro dos gastos com indivíduos sobreviventes ao longo de todo o período de análise, com uma taxa de crescimento também mais elevada (FIG. 2). A taxa de crescimento dos gastos per capita de 1995 a 2007 (em R\$ de 1995) é de cerca de 15\% para os mortos e aproximadamente $11 \%$ para os sobreviventes. 


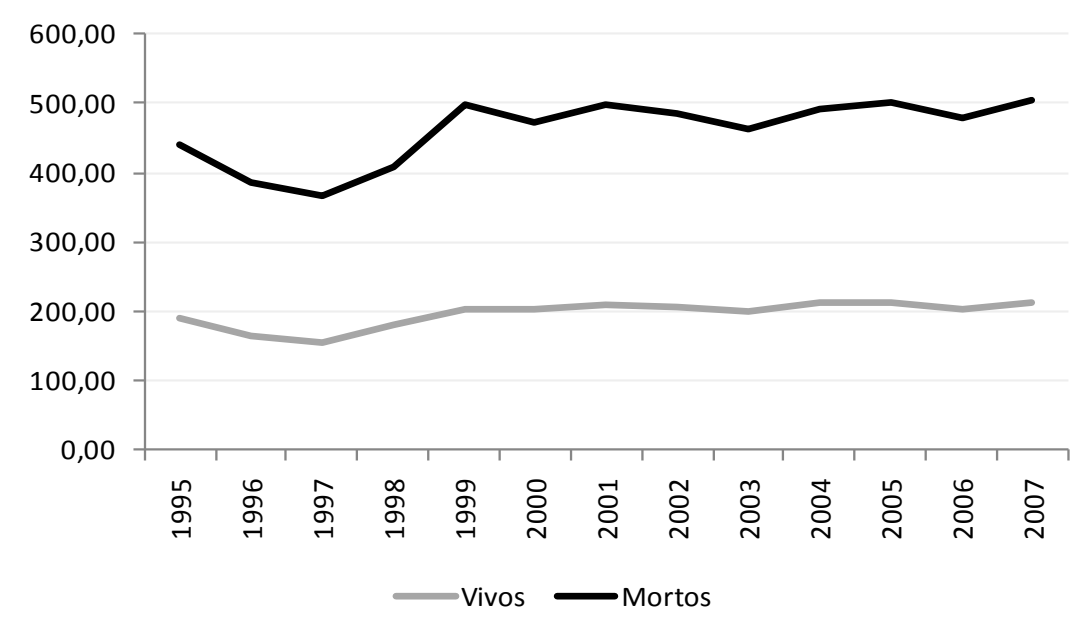

Figura 2 - Gasto per capita por status de sobrevivência (R\$ de 1995) - Brasil, 1995 a 2007

Fonte: DATASUS/SIH - 1995 a 2007.

Uma forma de verificar a importância relativa dos gastos relacionados à morte em relação ao gasto dos sobreviventes é por meio da razão de gastos. Isso é feito calculando-se a razão dos gastos totais relacionados à morte no ano de análise sobre os gastos dos sobreviventes. A FIG. 3 apresenta a razão de gastos para os gastos totais e os gastos per capita. Enquanto a razão de gastos total (mensurada pela razão do somatório de todos os gastos no ano dos mortos sobre os gastos totais de sobreviventes) apresenta uma elevação ao longo de todo o período, a razão de gastos per capita é relativamente constante, com aumento nos últimos períodos de análise. Como a razão de gastos totais mensura o gasto agregado, há um efeito composição que pode estar influenciando a tendência, como um aumento na população internada com saída por óbito (FIG. 1), que possui gastos mais elevados. Apesar disso, a razão de gastos é menor que um, indicando que o gasto agregado da população não sobrevivente é menor que o gasto agregado daqueles que permaneceram vivos. 
Total

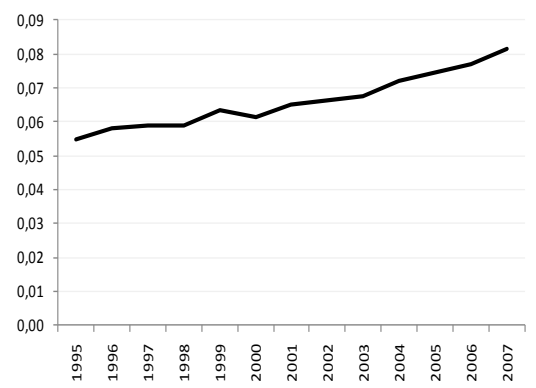

Per capita

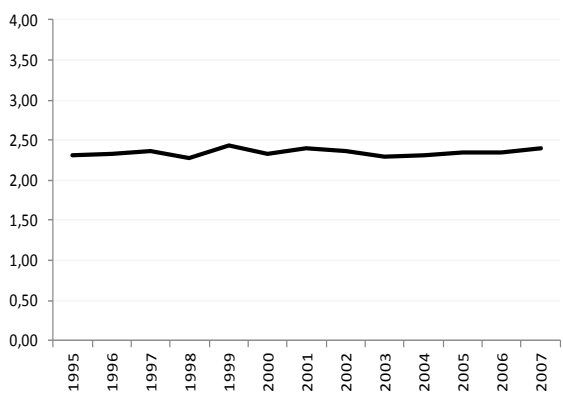

Figura 3 - Razão do gasto mortos/vivos (em R\$ de 1995) - Brasil, 1995 a 2007 Fonte: DATASUS/SIH - 1995 a 2007.

\subsection{Tendência dos Gastos por Grupo Etário}

A FIG. 4 apresenta o perfil etário dos gastos médios segundo status de sobrevivência em 1995, 2000 e 2007, em R $\$$ de 1995. A curva de gastos para os sobreviventes apresenta um formato de $J$ até o grupo etário de 60 a 64 anos, como é o formato típico da curva de gastos por grupo etário. Entre os sobreviventes, o maior gasto ocorre nas idades de 60 a 64 anos, quando então começa a decrescer. $\mathrm{O}$ gasto per capita foi maior em 2007 para os grupos de 15 a 24 anos e para os grupos etários quinquenais compreendidos entre as idades acima de 50 anos. No grupo etário de 60 a 64 anos, o crescimento do gasto médio de 1995 a 2007 foi de aproximadamente 15\%. No grupo de 80 anos e mais houve convergência dos gastos médios entre os três períodos analisados.

A figura dos gastos per capita para os pacientes terminais é distinta da encontrada para os sobreviventes, sendo decrescente entre os grupos etários e com maior variabilidade nos grupos etários mais jovens. Essa variabilidade é ocasionada pelo pequeno número de observações de pacientes que falecem nesses grupos. Nas idades mais avançadas o gasto diminui consideravelmente. Entre os períodos, os gastos foram superiores em 2007 para todos os grupos etários. 
Vivos

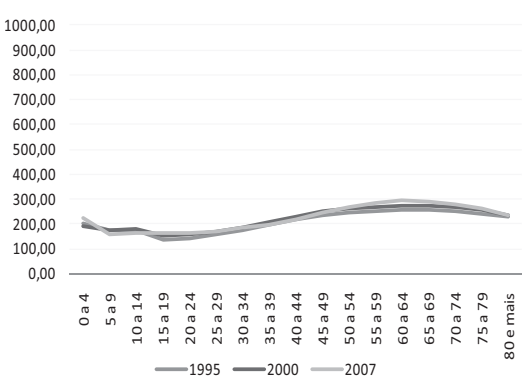

Mortos

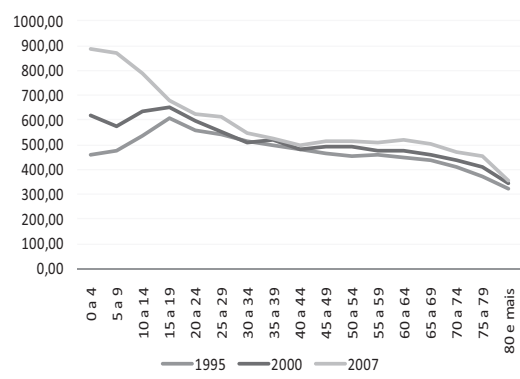

Figura 4 - Gasto per capita por status de sobrevivência e grupo etário (em R\$ de 1995) - Brasil, 1995, 2000 e 2007

Fonte: DATASUS/SIH - 1995 a 2007.

A razão de gastos entre mortos/vivos é apresentada na FIG. 5, para os gastos totais e per capita. Considerando apenas a razão de gastos totais, pode-se notar que há um crescimento nos gastos dos mortos em relação aos sobreviventes ao longo dos grupos etários para todos os anos, sendo esse crescimento maior em 2007. O aumento dessa razão de gastos com a idade e seu maior nível em 2007 refletem tanto a importância agregada dos gastos com mortes nos grupos etários mais velhos quanto um aumento na mortalidade hospitalar nos anos recentes.

Por outro lado, a razão de gastos per capita apresenta um padrão bem distinto do observado para os sobreviventes, com um comportamento decrescente entre os grupos etários. Isso indica a redução da diferença entre os gastos médios entre pacientes sobreviventes e terminais à medida que se envelhece. Os gastos com pacientes terminais são superiores em cerca de 4,5 vezes o gasto com pacientes sobreviventes no grupo de 10 a 14 anos, valor que cai para 1,5 no grupo de 80 anos e mais. Entre os anos analisados, a razão é praticamente coincidente, e a variabilidade nos primeiros grupos pode ser atribuída ao pequeno número de observações nesses grupos. A fim de comparar os resultados foi realizado um teste não paramétrico de médias de duas amostras independentes, pelo método Wilcoxon Mann-Whitney (Greene, 2000). Foram realizados dois tipos de comparações: (i) diferença nos gastos médios entre sobreviventes e mortos em cada grupo etário e ano; (ii) diferença nos gastos médios entre períodos 
(1995/2000, 2000/2007 e 1995/2007) para cada grupo etário e status de sobrevivência, separadamente. Os resultados mostraram que as únicas diferenças significativas entre gastos médios podem ser verificadas quando se comparam sobreviventes e não sobreviventes em cada grupo etário e cada ano (a 1\% de significância). A análise dos gastos médios entre dois períodos (1995/2000, 2000/2007 e 1995/2007) para cada grupo etário e status de sobrevivência não foi significativa. Isso significa que não se pode rejeitar estatisticamente a possibilidade de que os gastos médios dos períodos em cada grupo etário sejam iguais.

Total

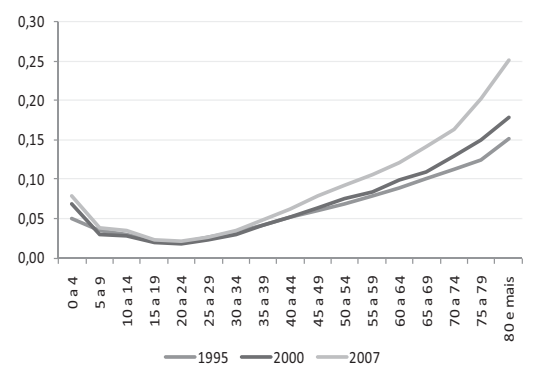

Per capita

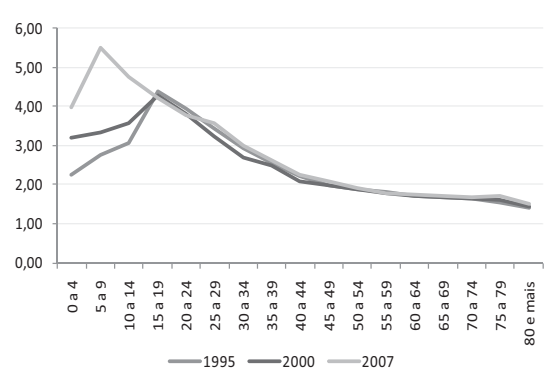

Figura 5 - Razão do gasto mortos/vivos (em R\$ de 1995) - Brasil, 1995 a 2007 Fonte: DATASUS/SIH - 1995 a 2007.

\section{Simulação do Gasto em 2050 por Status de Sobrevivência}

A distinção dos gastos pelo status de sobrevivência é muito útil para projeção de gastos com saúde. O conselho técnico do programa Medicare, nos Estados Unidos, tem sugerido a incorporação de tal desagregação nas projeções desde o ano 2000 (Gray, 2005), assim como a literatura atuarial sobre seguro saúde (Reese, 2000). A incerteza sobre o futuro dos gastos tem levado os autores a trabalharem com a suposição de gasto constante. Esse tipo de projeção é uma forma de avaliar qual seria o impacto demográfico puro sobre o futuro dos gastos com saúde (Gray, 2005). Pela importância da proximidade à morte para os gastos, os trabalhos passaram a realizar projeções de gastos constantes separados por status de sobrevivência (Cutler \& Sheiner, 1998). Neste caso, tanto os gastos quanto a população projetada devem estar desagregados por status de sobrevivência. 
Neste trabalho adotamos a mesma suposição dos trabalhos usualmente encontrados na literatura sobre o tema. A fim de incorporar o efeito do status de sobrevivência sobre os gastos com internações no Brasil em 2050, foram realizadas simulações dos gastos totais para este período, comparando a projeção baseada na multiplicação dos gastos médios pela população projetada e nos gastos médios separados por status de sobrevivência. Esse tipo de simulação representa uma abordagem contrafactual, isto é, o que aconteceria aos gastos variando apenas as características demográficas.

Para avaliar a projeção de gastos com internação, é necessário que se tenha a projeção do número de internações hospitalares. Essa projeção foi realizada considerando taxas de internação fixas em 2007 que, multiplicadas pelas projeções populacionais, ${ }^{3}$ fornecem o número esperado de internações no futuro (Finalyson, 2004). Como o objetivo do exercício é verificar a diferença no resultado da simulação quando os gastos por status de sobrevivência são incorporados, considerou-se que a taxa de internação na população por status de sobrevivência em 2050 seria a mesma daquela observada em 2007. Multiplicando então essa taxa de internação pelo número de pessoas projetado para 2050 pelo IBGE, revisão de 2008, obtém-se o número de internações para indivíduos sobreviventes e não sobreviventes, como mostra a Equação (1). A hipótese por trás desse tipo de projeção é que o único componente a variar no tempo corresponde à população, pois a relação entre internações/população permanece fixa no período (Rodrigues, 2010).

$$
I_{j}^{2050}=\sum_{i=x}^{x+n} \boldsymbol{T}_{i, j}^{2007} * P_{i}^{2050}
$$

Onde:

$I_{j}^{2050}=$ número de internações esperado em 2050 por status de sobrevivência

$T_{i, j}^{2007}=\frac{I_{i, j}^{2007}}{P_{i}^{2007}}=$ taxa de internação em 2007, na faixa etária i (de x anos a a $\mathrm{x}+\mathrm{n}$ anos) e status de sobrevivência $\mathrm{j}$. A taxa de internação é dada pela razão entre o número de internações em 2007 para indi-

3 As projeções populacionais utilizadas neste estudo correspondem àquelas elaboradas pelo Instituto Brasileiro de Geografia e Estatística (IBGE), revisão de 2008. 
víduos vivos ou mortos, definidos pelo subscrito $\mathrm{j}$, sobre a população total em 2007 na faixa etária i. $P_{i}^{2050}=$ população projetada em 2050 (pelo IBGE) na faixa etária i.

Após estimar o número de internações esperado em 2050 por status de sobrevivência, o passo seguinte consiste na simulação do gasto total em 2050, que combina a multiplicação do perfil etário dos gastos pelo número de internações projetado (Mahal \& Berman, 2001). A simulação dos gastos totais em 2050 foi realizada de duas formas: (i) pela multiplicação de gastos médios pelo número de internações projetado em 2050, como mostra a Equação (2); (ii) pela multiplicação dos gastos médios por status de sobrevivência pelo número de internações esperado por status de sobrevivência em 2050, conforme Equação (3):

$$
\begin{gathered}
G^{2050}=\sum_{i=x}^{x+n} G M_{i, j}^{2007} * I_{i, j}^{2050} \\
G^{2050}=\sum_{i=x}^{x+n} G M_{i, \text { sobreviventes }}^{2007} * I_{i, \text { sobreviventes }}^{2050}+\sum_{i=x}^{x+n} G M_{i, \text { mortos }}^{2007} * I_{i, \text { mortos }}^{2050}
\end{gathered}
$$

Onde:

$G^{2050}=$ gasto total projetado em 2050

$G M_{i, \text { sobreviventes }}^{2007}$ e $G M_{i, \text { mortos }}^{2007}=$ gasto médio na faixa etária i (de $\mathrm{x}$ anos a $\mathrm{x}+\mathrm{n}$ anos) com pacientes que sobreviveram em 2007 e com pacientes que faleceram em 2007, respectivamente, $I_{i, \text { sobreviventes }}^{2050} I_{i, \text { sobreviventes }}^{2050}=$ internações esperadas em 2050 para pacientes sobreviventes e pacientes que tiveram saída por óbito na faixa etária i.

As Equações (2) e (3) representam as simulações prospectivas, isto é, projeções do gasto total para 2050, considerando gastos médios fixos em 2007. A fim de analisar a sensibilidade dessas simulações, foram realizadas projeções retrospectivas para o ano de 2007. Nesta simulação, gastos médios foram mantidos fixos no ano de 1995 - similar às Equações (2) e (3) - e multiplicados pelo número esperado de internações em 2007, considerando a taxa de utilização em 1995, como na Equação (1). O objetivo da simulação retrospectiva é analisar a sensibilidade da projeção às hipóteses adotadas, principalmente 
em relação à diferença no resultado quando os gastos são desagregados por status de sobrevivência.

A TAB. 1 apresenta os resultados das simulações prospectivas do gasto total em 2050 e a variação no gasto entre 2007 e 2050, além de simulações retrospectivas. Considerando a simulação prospectiva, observa-se que os gastos totais simulados para 2050 variam consideravelmente quando é feita a distinção por status de sobrevivência. Em comparação aos gastos reais em 2007, seria observado um crescimento de cerca de $383 \%$ se fossem levados em consideração os gastos médios totais e $68 \%$ quando os gastos por status de sobrevivência são incorporados.

As simulações retrospectivas corroboram a tendência à superestimação do efeito demográfico sobre os gastos quando estes não levam em consideração os gastos associados à morte. Em relação ao gasto observado em 2007, as projeções tendo como base o ano de 1995 mostram um aumento de cerca de 42\%, considerando os gastos médios totais, e de 33\% quando os gastos levam em consideração os perfis de sobrevivência da população internada.

Essa diferença em relação ao gasto observado decorre das hipóteses adotadas, de gastos médios constantes e taxas de utilização constantes, que não condizem com o comportamento histórico das internações. Por este motivo não se pode dizer que o exercício realizado nessa seção se trata de uma projeção strictu sensu, e sim de uma simulação que tem como objetivo avaliar o efeito demográfico sobre o volume de gastos. No entanto, embora os dois resultados estejam distantes do gasto observado em 2007, esse exercício mostra que a distinção por status de sobrevivência leva a uma aproximação maior do gasto esperado do que quando essa opção não é introduzida na análise. 
Tabela 1 - Efeito do status de sobrevivência sobre o volume de gastos com internação em 2050 - Brasil

\begin{tabular}{|c|c|c|c|c|}
\hline \multirow[b]{2}{*}{ 1) } & \multirow[b]{2}{*}{ Simulação prospectiva } & \multicolumn{3}{|c|}{ Gasto total com internações (em R\$ de 1995) } \\
\hline & & $\begin{array}{l}\text { Observado em } 2007 \\
\text { (em bilhões de R\$) }\end{array}$ & $\begin{array}{l}\text { Esperado em } 2050 \\
\text { (em bilhões de R\$) }\end{array}$ & $\begin{array}{c}\text { Variação 2007/2050 } \\
\text { (em \%) }\end{array}$ \\
\hline A) & Gasto médio (Equação 2) & 2,38 & 11,52 & 383,6 \\
\hline B) & $\begin{array}{l}\text { Desagregado pelo status de } \\
\text { sobrevivência (Equação 3) }\end{array}$ & 2,38 & 4,00 & 68,3 \\
\hline 2) & Simulação retrospectiva & $\begin{array}{l}\text { Observado em } 2007 \text { (em } \\
\text { bilhões de R\$) }\end{array}$ & $\begin{array}{l}\text { Esperado em 2007* } \\
\text { (em bilhões de R\$) }\end{array}$ & $\begin{array}{c}\text { Variação 2007/2007* } \\
(\mathrm{em} \%)\end{array}$ \\
\hline A) & Gasto médio & 2,38 & 3,39 & 42,2 \\
\hline B) & $\begin{array}{l}\text { Desagregado pelo status de } \\
\text { sobrevivência }\end{array}$ & 2,38 & 3,17 & 33,2 \\
\hline
\end{tabular}

Fonte: DATASUS, estimativas populacionais, 1995 e 2007, SIH/SUS - 1995 e 2007; e Projeções Populacionais do IBGE, revisão 2008 - 2050.

Nota: o símbolo asterisco $\left({ }^{*}\right)$ representa as internações projetadas em 2007 considerando gastos fixos em 1995.

\section{Discussão}

O envelhecimento populacional tem sido colocado como um dos principais responsáveis pelo aumento nos gastos com saúde. Estimativas feitas para países desenvolvidos mostram que o efeito demográfico puro será responsável por uma parcela crescente do PIB no futuro, se consideramos o padrão etário dos gastos constante (Friedman, 2001; Ahn et al., 2005). Como bem documentado na literatura de economia da saúde, a curva de gastos por idade apresenta um perfil em $J$ (Nunes, 2004), indicando que os gastos tendem a crescer depois da infância, e com maior taxa de crescimento entre os idosos.

No entanto, a literatura aponta que o envelhecimento populacional pode não ser acompanhado por um aumento nos gastos totais, particularmente se o volume de gastos estiver concentrado no fim da vida, independente da idade à morte (Raitano, 2006). Uma possível explicação para isso é que há uma substituição de cuidado no fim da vida para os idosos, em direção aos cuidados de longa duração (long term care) ao invés do cuidado hospitalar. Este trabalho procurou mostrar como os gastos com internação variam por status de sobrevivência entre os grupos etários no Brasil e ao longo do tempo. Os resultados mostraram que a importância da morte no total de 
gastos com internação é evidente. $\mathrm{O}$ montante representa quase $8 \%$ do total de gastos, apesar de o percentual de mortes ser de pouco mais de $3 \%$. Além disso, o percentual de gastos relacionados à morte cresceu mais de $15 \%$ entre 1995 a 2007. Os idosos acima de 60 anos próximos à morte consumiram cerca de 2,3\% de todos os gastos com internações em 1995 e quase 4\% em 2007. Esse valor é superior à proporção de idosos próximos à morte na população, que corresponde a $1,13 \%$ e cerca de $2 \%$ da população total de internados em 1995 e 2007, respectivamente. A análise do padrão de gastos por grupo etário segundo status de sobrevivência mostrou que o padrão de gastos de sobreviventes e pacientes terminais é distinto. Enquanto o gasto de sobreviventes tende a crescer com a idade, o de mortos tende a diminuir consideravelmente, como encontrado na literatura internacional (Gabriele et al., 2006). Além disso, praticamente não houve alteração no padrão e nível desses gastos por grupo etário de 1995 a 2007. A combinação de redução dos gastos com não sobreviventes e aumento para os sobreviventes em relação à idade leva a uma pequena diferença entre sobreviventes e não sobreviventes nos grupos etários mais velhos.

Em relação à simulação dos gastos em 2050, observa-se que a incorporação de projeções desagregadas pelo status de sobrevivência dos pacientes internados gerou resultados muito distintos da projeção baseada em gastos médios totais. Ao manter os gastos per capita constantes no nível de 2007 multiplicados pelo número esperado de internações em 2050 levaria a um aumento real de aproximadamente 9\% ao ano dos gastos totais em 2050 em função, exclusivamente, do envelhecimento populacional (o acumulado no período de 2007 a 2050 seria de quase 384\%). Ao desagregar os gastos médios pelo status de sobrevivência e multiplicá-los pelo número de internações esperadas em 2050 para pacientes sobreviventes e pacientes terminais, esse crescimento seria de apenas $1,6 \%$ ao ano. Simulações retrospectivas para 2007, considerando cenário de gastos e utilização constantes em 1995, mostraram que a não incorporação de gastos médios desagregados pelo perfil de sobrevivência leva a uma superestimação do efeito demográfico sobre o montante de gastos com internação. Resultado semelhante foi encontrado para a população beneficiária do Medicare, nos Estados Unidos, em 2050 (Cutler \& Sheiner, 1998). Utilizando a mesma metodologia do presente traba1ho, os autores estimaram um crescimento de cerca de $9 \%$ ao ano em termos reais nos gastos com o Medicare em 2050, sem levar em 
consideração informações desagregadas pelo status de sobrevivência e cerca de $2 \%$ quando essas informações são incorporadas. Dessa forma, aliar dados sobre gastos com pacientes sobreviventes e terminais às projeções possibilita análises mais refinadas sobre mudanças nos gastos associadas a melhorias na mortalidade (Gray, 2005).

Os gastos com pacientes que não sobrevivem decorrem da interação de vários fatores que afetam o padrão de gastos por grupo etário, como diferentes causas de doenças, distintas taxas de internação e tempo médio de internação, e complexidade dos procedimentos, que costumam ser mais agressivos em idades mais novas - e, muitas vezes, mais caros - do que em idades mais avançadas (Raitano, 2006).

Essa é uma limitação desse exercício que merece ser investigada com mais detalhes. Evidências para países desenvolvidos mostram que está havendo uma compressão da morbidade para idades mais avançadas, o que pode explicar, em parte, essa redução dos gastos médios com pacientes terminais (Fries, 2003; Fogel, 2005).

Uma limitação do trabalho refere-se à forma como é mensurado o gasto por status de sobrevivência. Como não é possível acompanhar a história de cada paciente ao longo do período de um ano com o banco de dados utilizado, um mesmo paciente pode ter sido internado mais de uma vez no período de análise, com motivos distintos de saída do hospital. Se um paciente for internado duas vezes, sendo na primeira saída por alta e na segunda vez saída por óbito, na primeira internação o gasto será computado como um gasto de sobrevivente, e na segunda um gasto de não sobrevivente. Como os gastos com internação para pacientes no último ano de vida são muito altos e a probabilidade de morte é maior em idades mais avançadas, isso poderia explicar a redução da diferença na razão de gastos vivos/mortos nos últimos grupos etários. O elevado gasto em idades mais avançadas nos sobreviventes pode refletir o fato de que ele está próximo à morte e, por isso, incorrendo em altos custos. No entanto, mesmo os trabalhos que analisam os gastos em uma base de comparação anual encontram razões de gastos decrescentes nos últimos grupos etários (Payne, 2009; Maia, 2012).

Uma segunda limitação do trabalho refere-se à análise de gastos apenas com internações. Para aprofundar o entendimento de como os gastos variam por grupo etário segundo o status de sobrevivên- 
cia, deveríamos incluir outros itens de gasto com saúde. Evidências apontam que os gastos com cuidados de longa duração no último ano de vida aumentam com a idade (Raitano, 2006). Dessa forma, a redução na razão dos gastos mortos/sobreviventes em relação aos gastos hospitalares, como mostrado nesse trabalho e em outros para vários países (Lubitz et al., 1995; McGrail et al., 2000; Ahn et al., 2005) pode ser parcialmente compensada pelo aumento nos gastos com cuidados domiciliares ou outros tipos de cuidado que não os hospitalares.

Para Weaver et al. (2009), o aumento da demanda por cuidados de longa duração provoca um efeito sobre o tipo de suporte (formal ou informal) dependendo do grau em que a utilização de serviços de saúde está concentrada no fim da vida. Os autores apresentam evidências de que quanto maior a proximidade à morte, maior também é a probabilidade de demandar cuidados permanentes. Porém, a disponibilidade de suporte informal reduz a necessidade de institucionalização do cuidado. Ser casado, por exemplo, reduz a probabilidade de demanda por cuidados de longo prazo no mercado formal, pois os cônjuges agiriam como cuidadores de seus parceiros.

Segundo Evans et al. (2004) citado por Gabriele et al. (2006), as razões pelas quais responsabilizar o envelhecimento populacional são várias. Por um lado pode ser um artefato político para distrair a atenção de aumentos nos gastos ocasionados por outros fatores, como a elevação dos custos no setor saúde. Além disso, fortalece a justificativa de ampliação do financiamento via mix público-privado. Simulações para 2050 mostram que o efeito do envelhecimento sobre os gastos totais com internações tende a diminuir consideravelmente se ponderamos os gastos médios pelo status de sobrevivência. Um fator que poderia reduzir então a pressão dos gastos no futuro é a redução da mortalidade, que faz com que existam menos pessoas em seu último ano de vida. No entanto, o efeito final do declínio da mortalidade sobre o perfil etário de gastos com saúde depende, em grande parte, do efeito da morbidade. 


\section{Considerações Finais}

Em linhas gerais, o trabalho mostrou que é possível que o envelhecimento populacional não seja tão responsável por um aumento nos gastos com saúde como atualmente é discutido. A maior parte dos gastos está concentrada no fim da vida e, caso a esperança de vida continue a aumentar, existirão menos pessoas em idades próximas à morte no futuro. Esse efeito quantitativo poderia levar a um arrefecimento nos gastos com saúde em função do envelhecimento, como demonstrado no exercício sobre simulação dos gastos com internações em 2050.

Do ponto de vista macroeconômico e da formulação de políticas públicas na área de saúde, a distinção dos gastos com saúde por status de sobrevivência é um instrumental útil nas projeções de gastos. Espera-se que os gastos cresçam em menor proporção do que o esperado quando se considera o gasto próximo à morte. Do ponto de visa microeconômico, a distância à morte é um fator importante para explicar a concentração do gasto em determinados grupos populacionais. Identificar e analisar tratamentos para patologias terminais seria uma forma de promover práticas mais custo-efetivas.

\section{Referências}

AHN, N.; Garcia, J.R.; Herce, J. A. Demographic uncertainty and health care expenditure in Spain. Documento de Trabajo 2005-07, 2005.

ANDRADE, M. V.; Maia, A. C.; Rodrigues, C. G. Gastos com serviços médicos por status de sobrevivência no setor de saúde suplementar no Brasil. Revista Brasileira de Estudos de População (no prelo), 2012.

ANTOLIN, P.; Suyker, W. How Should Norway Respond to Ageing?, OECD Economics Department Working Papers, No. 296, OECD Publishing. Disponível em: <http://dx.doi. org/10.1787/385156551776>, 2001.

BATLJAN, I.; Lagergren, M. Inpatient / outpatient health care costs and remaining years of life - effect of decreasing mortality on future acute health care demand. Social Science and Medicine, vol. 59, p. 2459-2466, 2004.

BERENSTEIN, C.K. Os efeitos de idade e proximidade à morte sobre os gastos com internações no SUS: evidências com base no caso de Minas Gerais, 2004/2005. 2009. Tese de Doutorado (Doutorado em Demografia). Cedeplar, UFMG, Belo Horizonte, 2009, 95 p.

BOGUE, R.; Hall, C.H.; La Forgia, G.M. Hospital Governance in Latin America: results from a four nation survey. Washington: The World Bank Health, Nutrition and Population (HNP), 2007. Discussion Paper. Disponivel em: www.siteresources.worldbank.org/HEALTHNUTRITIONANDPOPULATION/Resources/291627-1095698140167/LACHospitalGovernanceFinal.pdf>. 
BREYER, F.; Felder, S. Life expectancy and health care expenditures: a new calculation for Germany using the costs of dying. Health Policy, vol. 75, p. 178-186, 2006.

CALVER, J.; Bulsara, M.; Boldy, D. In-patient hospital use in the last years of life: a Western Australian population-based study. Aust N Z J Public Health, vol. 30, p.143-146, 2006.

CUTLER, D. M.; Sheiner, L. Demographics and Medical Care Spending: Standard and non-standard effects. Working Paper 6866. Cambridge, MA.: National Bureau of Economic Research, 1998.

DANG, T.; Antolin, P.; Oxley, H. Fiscal Implications of Ageing: Projections of age-related spending. Economics department working papers no. 305, ECO/WKP(2001)31. Paris: Organization for Economic Co-operation and Development, 2001.

FINLAYSON, G.S. et al. Anticipating change: how many acute care hospital beds will Manitoba regions NEED IN 2020? CANADIAN JOURNAL ON AGING, [CAMBRIDGE], V. 24, P. 133-140, SEP.2004. SUPPL. 1.

FOGEL, R.W. Changes in the physiology of aging during the twentieth century. Cambridge: National Bureau of Economic Research, 2005. (NBER Working Paper, 11233).

Fogel, R.W. Changes in the process of aging during the twentieth century: findings and procedures of the early indicators project. Cambridge: National Bureau of Economic Research, 2003. (NBER Working Paper, 9941).

FOGEL, R.W. Forecasting the cost of U.S. health care in 2040. Nber Working Papers, $\mathrm{n}^{\circ}$ 14361, Cambridge, 2008.

FRIES, J.F. Compression of morbidity in the elderly. Vaccine, Kidlinton, v. 18, p. 1584-1589. 2000.

FRIES, J.F. Measuring and monitoring success in compressing morbidity. Ann Intern. Med., Philadelphia, v. 139, p. 455-459. 2003.

FRIEDMAN, J.N. Predicting Medicare Cost Growth. In: Improving Health Care Cost Projections for the Medicare Population: Summary of a Workshop http://www.nap.edu/catalog/12985.html, 2001.

GABRIELE, S.; Cislaghi, C.; Constantini, F.; Innocenti, F.; Lepore, V.; Tediosi, F.; Valerio, M.; Zocchetti, C. Demographic factors and health expenditure profiles by age: the case of Italy. ENEPRI Research Report, $\mathrm{n}^{\circ}$ 18, Maio, 2006.

GRAY, A. Population ageing and health care expenditure. Ageing Horizons, vol. 2, p.15-20, 2005.

GREENE, W.H. Econometric Analysis. 4th ed. New Jersey: Prentice-Hall, 2000. 1.004 p.

KILSZTAJN, S. et al. Serviços de saúde, gastos e envelhecimento da população brasileira. Revista Brasileira de Estudos da População, Campinas, v. 20, n.1, p. 93-108, jun. 2003.

Lakdawalla, D.; Philipson, T. The rise in old-age longevity and the market for long-term care. The American Economic Review, Nashville, v. 92, n. 1, p. 295-306, Mar. 2002.

LAYTE, R. Na analysis of the impacto f age and proximity of death on health care costs in Ireland. ESRI Working Paper n 193, 2007.

LEE, R. \& Miller, T. An approach to forecasting health expenditures, with application to the U.S. Medicare System. Health Services Research, vol. 37(5): 1365-1386, 2002.

LUBITZ, J.D.; Riley, G.F. Trends in Medicare payments in the last year of life. The New England Journal of Medicine, vol. 328(15): 1092-1096, 1993.

LUBITZ, J.; Beebe, J.; Baker, C. Longevity and Medicare expenditures. The New England Journal of Medicine, vol. 332(15): 999-1003, 1995.

MAHAL, A.; Berman, P. Health expenditures and the elderly: a survey of issues in forecasting, methods used, and relevance for developing countries. The Global Burden of Disease 2000 in Aging Populations. Research Paper $n^{\circ}$ 01/23, 2001.

MCGRAIL, K.; GREEN, B.; BARER, M.L.; EVANS, R.G.; Hertzman, C.; Normand, C. Age, cost of acute and long-term care and proximity to death: evidence for 1987-88 and 1994-95 in the British Columbia. Age and Ageing, vol. 29: 249-253, 2000. 
MAYHEW, L. Health and elderly care expenditure in and aging world. International Institute for Applied Systems Analysis, Laxenburg, Austria. Research Report, 2000.

MAIA, A.C.; Andrade, M.V.; Chein, F. Estudo longitudinal do efeito da idade e tempo até a morte em gastos com saúde. Mimeo. 2012.

MILLER, T. Increasing longevity and medicare expenditures. Demography, vol. 38(2): 215-226, 2001.

NUNES, A. O envelhecimento populacional e as despesas do Sistema Único de Saúde. In: CAMARANO, A.A. (Org.). Os novos idosos brasileiros: muito além dos 60? Rio de Janeiro: IPEA, 2004.

OECD. Projecting OECD health and long-term care expenditures: what are the main drivers? Economics Department Working Papers $n^{\circ}$ 477, 2005.

PAYNE, G.; Laporte, A.; Deber, R.; Coyte, P.C. Counting backward to health's care future: using time to death modeling to identify changes in the end-of-life morbidity, and the impact of aging on health care expenditures. The Milbank Quarterly, vol. 85(2): 213-257, 2007.

PAYNE, G.; Laporte, A.; Foot, D.K.; Coyte, D.C. Temporal trends in the relative cost of dying: evidence from Canada. Health Policy, vol. 90: 270-276, 2009.

POLDER, J.J.; Achterberg, P.W. Cost of illness in the Netherlands. Centre for Public Health Forecasting. 2004. Disponível em: < http://www.rivm.nl/kostenvanziekten/downloads/kvzrapport/ Cost_of_illness_highlights.pdf $>$.

POLDER, J.J.; Barendregt, J.J.; Oers, H.V. Health care costs in the last year of life - the Dutch experience. Social Science \& Medicine, vol. 63: 1720-1731, 2006.

RAITANO, M. The impact of death-related costs on health care expenditure: a survey. ENEPRI Research Report $\mathrm{n}^{\mathrm{o}} 17,2006$.

REESE, A.J. Development of the Last-Year-of-Life Valuation Model for Retiree Medical Plans. North American Actuarial Journal, 4(2): 116-127, 2000.

RODRIGUES, C.G. Dinâmica demográfica e internações hospitalares: uma visão prospectiva para o Sistema Único de Saúde (SUS) em Minas Gerais, 2007 a 2050. 2010. 249 p. Tese (Doutorado em Demografia) - Centro de Desenvolvimento e Planejamento Regional, Universidade Federal de Minas Gerais, Belo Horizonte, 2010.

SCITOVSKY, A.A. "The high cost of dying": what do the data show? The Milbank Memorial Fund Quarterly: Health and Society, vol. 62(4):591-608, 1984.

SESHAMANI, M.; Gray, A.M. A longitudinal study of the effects of age and time to death on hospital costs. Journal of Health Economics, vol. 23, p. 217-235, 2004.

SPILLMAN, B.C.; Lubitz, J. The effect of longevity on spending for acute and long-term care. The New England Journal of Medicine, vol. 342, n. 19: 1409-1415, 2000.

STEARNS, S.; Norton, E. Time to include time to death? The future of health care expenditures predictions. Health Economics, vol. 13(4): 315-327, 2004.

WEAVER, F.; Stearns, S.C.; Norton, E.C.; Spector, W. Proximity to death and participation in the longterm care market. Health Economics, Chichester, v. 18, n. 8, p. 867-883, Aug. 2009.

YANG, Z.; Norton, E.C.; Stearns, S.C. Longevity and health care expenditures: the real reasons older people spend more. The Journals of Gerontology, vol. 58B(1): S2-S10, 2003.

ZWEIFEL, P.; Felder, S.; Meier, M. Ageing of population and health care expenditure: a red herring? Health Economics, vol. 8: 485-496, 1999. 